\section{Cahiers de Narratologie}

Analyse et théorie narratives

$19 \mid 2010$

Images composites, arts pluriels

\title{
Cinéma, peinture et numérique : hybridité de l'image chez Peter Greenaway
}

\section{Fabien Maheu}

\section{(2) OpenEdition}

\section{Journals}

Édition électronique

URL : http://journals.openedition.org/narratologie/6177

DOI : 10.4000/narratologie.6177

ISSN : 1765-307X

Éditeur

LIRCES

\section{Référence électronique}

Fabien Maheu, «Cinéma, peinture et numérique : hybridité de l'image chez Peter Greenaway », Cahiers de Narratologie [En ligne], 19 | 2010, mis en ligne le 22 décembre 2010, consulté le 10 décembre 2020. URL : http://journals.openedition.org/narratologie/6177; DOI : https://doi.org/10.4000/narratologie. 6177

Ce document a été généré automatiquement le 10 décembre 2020.

\section{c) (†) $९$}

Cahiers de Narratologie - Analyse et théorie narratives est mis à disposition selon les termes de la licence Creative Commons Attribution - Pas d'Utilisation Commerciale - Pas de Modification 4.0 International. 


\title{
Cinéma, peinture et numérique : hybridité de l'image chez Peter Greenaway
}

\author{
Fabien Maheu
}

1 Depuis le début des années quatre-vingt, Peter Greenaway n'a cessé de naviguer d'un monde à l'autre: de la pratique industrielle du cinéma au marché élitiste des arts plastiques. Cette réalité contextuelle se double d'une ambition esthétique, qui est de nourrir le cinéma, compris comme un art mineur n'ayant pas trouvé son langage propre, des immenses richesses formelles explorées par la peinture depuis plus d'un millénaire.

2 De toute évidence, cette recherche d'un cinéma plastique, déjà sensible dans ses courts métrages et parfois dans les longs, trouve dans l'avènement du numérique un terrain d'épanouissement sans précédent. Le réalisateur est-il un précurseur qui n'attendait que l'outil approprié à de visionnaires projets, ou appartient-il simplement à une génération d'artistes qui, plus que jamais au courant des dernières innovations et profitant de l'accessibilité accrue des matériels de pointe percevait intuitivement les directions à venir que la technologie allait imprimer à l'esthétique? On ne peut s'empêcher de penser à Steve Reich, composant dès les années soixante la pièce Slow Motion Sound, censée être constituée d'un enregistrement ralenti plusieurs dizaines de fois sans modification de tonalité. Les moyens techniques ne permettant pas la réalisation effective de la pièce, le compositeur se contenta de la décrire en une phrase succincte, pour finalement en réaliser le principe, une vingtaine d'années plus tard, à l'aide des technologies numériques.

3 Dans le cas de Peter Greenaway, ce sont les premières possibilités de transfert d'image analogique / numérique mis au point au Japon à la fin des années quatre-vingt qui permirent au réalisateur de découvrir son langage audiovisuel le plus pertinent, celui qui répond le mieux au désir d'hybridité que son proto-cinéma manifestait déjà clairement. Invité dans le cadre d'un programme de production et de développement initié notamment par la NHK (la chaîne culturelle japonaise), Greenaway put tester en 
compagnie de l'infographiste Eve Ramboz, les nouvelles possibilités de compositing qui donnèrent finalement naissance à Prospero's Books, œuvre majeure et pourtant mal connue.

4 Ce sont bien entendu les nouvelles possibilités du numérique, et en l'occurrence de la haute définition, qui rendirent possible cette avancée du cinéma comme médium avant tout plastique : les outils de l'infographie permirent au réalisateur-peintre des opérations de collage auparavant trop onéreuses et trop lourdes, et ce, avec la possibilité de revenir en arrière, d'essayer, de tester, de choisir. Le cinéma numérique comme matériau plastique était né.

5 Pour autant, le concept de «numérique » demande à être précisé, tant il est vrai qu'il reste un réceptacle fantasmatique fort dans les élites culturelles, et que par ailleurs l'approche du phénomène numérique par le réalisateur britannique évolue dans le courant de sa carrière.

6 Il existe plusieurs réalités recouvertes par le terme « numérique ». Citons-en trois, parmi les plus usitées. La première, qui répond le mieux au terme qui la désigne, est en fait un art basé sur la programmation, un «art du code» en somme. Pour composer cet art comme pour le lire, il faut être capable de programmer, c'est-à-dire d'écrire un logiciel. Cet «art du code» se rencontre essentiellement dans les sphères discrètes de l'art contemporain. La seconde acception du terme désigne en fait l'ergonomie des outils informatiques traitant le son et l'image: palettes graphiques, logiciels de traitement audio, outils de montage et de compositing vidéo. Ce sont ces outils qui intéresseront tout d'abord Greenaway, car ils permettent de produire du matériel enregistré ou fixé, comme le sont le cinéma et la peinture. En troisième lieu vient ce qu'il est convenu d'appeler le traitement en «temps réel » ou même le "génératif ». Dans ce cas, l'informatique ne se contente pas de traiter une information après l'autre et d'enregistrer le résultat dans des fichiers disponibles pour la relecture. L'accent est mis au contraire sur le traitement immédiat et la transformation des ces informations en signaux qui paraissent simultanés. Le but n'est plus l'enregistrement mais la délivrance immédiate d'une réponse à des stimuli et ce, sans délai. Greenaway s'intéresse aujourd'hui à ces technologies essentiellement sous deux formes: d'une part la performance de Vjing réalisée dans les clubs à partir de matériaux tirés des rushes des Tulse Luper Suitcases et d'autre part Second Life qui représente pour lui le terrain d'expression privilégié des artistes de demain.

7 Si le réalisateur britannique est historiquement aux avant-postes de l'intégration du numérique au cinéma narratif, il ne fait qu'annoncer un mouvement global et partagé. Graduellement, les cinéastes intègrent l'ergonomie «infographique » à leurs écritures audiovisuelles, explorant notamment de nouvelles configurations du rapport espacetemps. La démultiplication des possibilités d'écriture visuelle entraîne également la prise en compte de la dimension verticale de l'image, c'est-à-dire de sa profondeur virtuelle dans un espace initialement plan, compris non plus comme le lieu privilégié de la captation photographique, mais comme un " canevas "plastique sur lequel sont réalisées des compositions d'éléments "photoscopiques » et plastiques. Le spectateur est bien entendu amené à adopter une lecture complexe du tissage des informations sur l'espace disponible du canevas. Cette ergonomie favorise une fragmentation croissante de l'œuvre et peut induire de nouveaux modes d'organisation des matériaux, au-delà même de la relation image-son, au delà du cinéma. L'œuvre devient alors un composite transversal usant de tous les médias disponible et utiles à son développement : cinéma, littérature, travaux plastiques, Internet, etc. Le récent opus The Tulse Luper Suitcases (de Peter 
Greenaway) est un exemple de ce redéploiement des ressources médiatiques au service de l'œuvre narrative.

8 De nombreux films de fiction grand public utilisent aujourd'hui le traitement numérique de l'image de manière totalement transparente et certaines caractéristiques initiales du cinéma s'en voient bouleversées. Dans le champ d'application des nouvelles images numériques, le photogramme, comme unité minimale photographique, tend à disparaître. La photographie se voit alors dépossédée de sa graphie: il ne s'agit plus en effet que de photoscopie. Les signaux résultant de la captation ne donnent plus lieu à une inscription sous forme d'images-traces, mais sous forme de données chiffrées. A mesure que son support physique disparait, l'image gagne en qualités liées à l'abstraction. Elle est alors capable de s'actualiser de multiples manières, autant de fois que souhaité et sur des supports de natures très différentes (moniteur, impression, etc.). Ainsi, de nombreux produits audiovisuels intègrent-ils des images de provenance et de qualité diverses dont la numérisation favorise la mixité, pour les actualiser dans un même et unique écran .

Cette nouvelle forme d'image comporte plusieurs niveaux de réalité. L'actualisation est précédée d'une zone de modélisation, elle-même subdivisée en deux objets distincts. Tout d'abord, le modèle en lui-même, qui peut être en trois dimensions, agencement d'un maillage et de textures, ou bien, dans le cas d'une facture bidimensionnelle, un empilement de calques superposés. Dans un second temps seulement intervient le rendu de ce modèle, c'est-à-dire le calcul d'un point de vue et d'un état donné du modèle et qui donnera lieu à une représentation possiblement visible de l'image. L'actualisation, quant à elle, se fait à partir du calcul du rendu, par un moniteur, un support vidéo, un tirage papier ou tout autre moyen choisi. Si l'on voulait artificiellement établir une relation entre la génération d'images numériques et la captation d'images photographiques, le modèle tiendrait le rôle de profilmique, le rendu celui de négatif, et l'actualisation, celui de pellicule projetée sur un écran.

10 Or, si dans l'ergonomie analogique liée à la pellicule de celluloïd, l'opération plastique la plus répandue reste l'étalonnage (stade du négatif), dans la pratique du cinéma numérique, les interventions sont rendues possibles et simplifiées, non plus seulement au stade du rendu, mais surtout dans la sphère du modèle. Dans le cas d'un traitement bidimensionnel, ces opérations ne relèvent plus de trucages optiques coûteux et complexes et ne sont plus le résultat d'une commande passée à un studio d'effets spéciaux. Elles font maintenant partie de la palette d'outil du monteur qui peut les exécuter au moyen d'un matériel simple et économiquement abordable. Dans une pratique contemporaine, cette nouvelle ergonomie du montage favorise l'utilisation du compositing comme fait grammatical naturalisé.

11 Dans le contexte traditionnel du cinéma analogique, l'image est inféodée aux capacités techniques de l'appareil d'enregistrement et aux conditions de projection, le cadrage étant assuré exclusivement à l'étape du filmage. Toute intervention ultérieure sur le cadre ou sur le ratio de l'image est perçue comme une défiguration imputable aux nécessités industrielles et notamment à celles de la diffusion hertzienne. A l'inverse, la révolution numérique permet à l'image de se penser indépendamment de ses particularités originelles de format.

12 Dès le premier quart du siècle, la forme narrative du long métrage tend à se figer et, avec elle, le ratio plein cadre de l'écran se fait espace naturaliste. Les divers caches ainsi que les cartons utilisés par le cinéma des premiers temps disparaissent, privant peu à peu l'écran de sa dimension plastique pour en faire un mime conventionnel du regard de la caméra. 
Si les années soixante-dix remettent à l'honneur pour un temps le split-screen, il faut attendre les années quatre-vingt et l'explosion des films institutionnels à bas prix réalisés en vidéo pour que se naturalisent peu à peu les vignettes et les images multi-écrans. C'est enfin l'habillage télévisuel des années quatre-vingt dix qui proposera la forme, désormais standard, des écrans multiples sur fond noir qui, peu à peu, prennent possession des écrans de cinéma.

13 Se saisissant de cette esthétique nouvelle, les jeunes réalisateurs tentent d'inventer des narrations capables d'intégrer les écrans multiples et de se les approprier. Ce n'est souvent pas chose facile. Notons les expérimentations passionnantes de Michel Gondry qui, tout en se pliant aux exigences rythmiques et de format du vidéo-clip musical, propose des modes narratifs originaux tout en développant des techniques de coprésence inédites. Dans ses travaux, la question de la naturalisation du collage se pose en permanence et semble essentiellement ne pas vouloir se résoudre.

14 Chez Peter Grenaway en revanche, le choix de l'hétérogénéité est radical. Dès les premières images de son cinéma structural, le rapport d'hybridité se joue dans la relation avec la peinture, référent permanent et définitif de sa pratique de l'image. Il convient donc d'aborder les matériaux produits par le réalisateur anglais avec absence de préjugé formel, et de traiter sur le même plan et pour les mêmes axes de recherche à la fois les films et les diverses œuvres plastiques qui n'ont cessé d'être produites depuis les années soixante. On trouve donc dans les peintures des effets de la pratique du cinéma et on rencontre fréquemment dans les films des tentatives pour répondre à des questions picturales.

15 Mais tout d'abord, la picturalité s'exprime de manière explicite dans les œuvres sur pellicule. L'hommage à l'histoire de l'art s'effectue parfois de manière littérale, sous forme de transsubstantiations aisément repérables, telle cette reconstitution d'une leçon d'anatomie à la manière de Rembrandt dans le palais de Prospero (Prospero's Books), opérant la synthèse de deux tableaux du maître hollandais : La Leçon d'anatomie du docteur Dayman pour la composition regroupant l'anatomiste et ses disciples autour du corps disséqué et un détail de Nicolaes Tulp pour la vue en raccourci du corps, à la manière d'un Mantegna. Autre référence explicite, celle faite au banquet de Franz Hall qui inspire la dégaine de la compagnie d'Albert Spica dans The Cook, the Thief, his Wife and her Lover. Une copie du tableau est exposée à l'arrière plan de la salle à manger où le voleur prend quotidiennement ses repas. La référence est donc intradiégétique et consentie sinon par les malfrats, au moins par le décorateur de la salle à manger. Le dessinateur Neville (The Draughtsman's contract) est semble-t-il lui aussi enclin à ce type de mise en perspective. Désignant une peinture de Van Dick intitulée Hommage à l'optique, le jeune homme en propose une lecture parabolique qui implique et explique maladroitement le quotidien au château de Mme Herbert. Ici, la dramaturgie de la peinture se superpose à celle du film.

16 Bien souvent, le traitement de l'image photographique produit des résultats proche d'un travail de peinture. Dans $H$ is for House, de nombreux plans sont délibérément composés à la manière d'une nature morte, et Intevalls qui feint de montrer Venise, ne fait qu'aligner des surfaces plates de murs sur lesquels s'étalent des lambeaux d'affiches déchirées. De Venise nous ne voyons ni les canaux, ni les ponts, ni rien de ce qui fait le cachet de la ville, laquelle est niée dans sa spécificité au profit de ses surfaces pariétales, plastiques et bornées par le cadre de la prise de vue. Greenaway fait ici de la ville du carnaval un livre de peinture moderne radicale. A la manière de la photographie picturale ou du Land Art, le réalisateur n'hésite d'ailleurs pas à dénaturer le paysage à des fins plastiques, comme 
lors de cette vue abstraite de l'horizon marin dans The Falls, photographié le diaphragme presque clos, faisant de la mer et du ciel une matière noire sans contour et ne laissant éclater que la myriade de reflets produits par la présence du soleil au dessus des flots.

17 Avant même de bénéficier de plates-formes de traitement numérique, on voit combien les possibilités plastiques de l'image vidéo sont exploitées dans L'Inferno, adapté de Dante et co-réalisé avec le peintre Tom Phillips. La représentation de Cerbère y est réalisée au moyen d'un effet de traçage par retard d'affichage. Les points les plus lumineux de l'image produisent donc des traces qui resteront affichées de manière indélébile sur l'image. Le visage du comédien représentant le gardien des Enfers se voit déformé à la manière d'un Bacon et semble finalement acquérir les deux gueules qui manquaient à sa représentation pro-mythologique.

18 A Zed and two Nought est empli de collages que l'on pourrait qualifier de «situationnels ». Il s'agit de compositions de l'image, parfois fixe, mais qui parfois ne résistent pas au mouvement de la caméra, dans lesquelles on détecte à l'évidence un agencement esthétique proche de tel ou tel mouvement pictural, ou provoquant un effet de sens visuel fort. C'est le cas lors du plan fixe surplombant de la table de composition photographique d'oliver alors que celui-ci, effondré, pleure la disparition de sa femme. La nature morte composée de pellicules, de diapositives et d'objectifs en contre-jour sur fond de papier millimétré devient un collage à l'esthétique pop. A quelques plans de là, le jeu de perspective visuelle élaboré autour de l'accident de voiture fait ironiquement sortir le tigre imprimé sur l'affiche d'arrière plan, hors du capot de la voiture immobilisée, indiquant en quelque sorte que le moteur est en train de rendre l'âme.

19 Greenaway met en place des circulations entre cinéma et peinture qui parfois prennent l'aspect de concurrences. Un certain nombre de travaux pastiques ont été recyclés pour l'écran, d'autres ont été spécifiquement réalisés pour être filmés. Mais dans tous les cas, le filmage de surfaces peintes est toujours générateur de tensions productives et de questionnements sur les médias.

20 Vertical feature remakes propose comme matière visuelle principale des documents présentés comme des notes, plans et diagrammes réalisés par le protagoniste absent Tulse Luper. Bien entendu, l'inadéquation entre cette annonce et les objets qui nous sont donnés à voir induisent une forme de « lâcher prise » de la part du regardeur et ouvrent la possibilité de regarder les écrans successifs comme des évocations ressortant de la poésie visuelle ou de l'expérimentation plastique. Le statut de ces objets est indécidable : sont-ce des documents intradiégétiques ou constituent-ils une matière de représentation à part entière qui se serait substituée à la photographie du profilmique?

21 Parallèlement à la réalisation d'un long métrage, Greenaway réalise souvent des travaux plastiques en relation avec les questions esthétiques que soulève le film. Ces travaux sont produits avant, mais aussi pendant et après les opérations de fabrication cinématographiques. Ce sont, de l'aveu du réalisateur, des moyens de répondre à des questions irrésolues, ou d'aborder différemment les enjeux soulevés lors du tournage par exemple. Si ces productions ont une existence propre et autonome, elles n'en restent pas moins reliées au film source et forment avec lui une entité commune et intermédiale.

Dans The Pillow Book, Greenaway tente d'abstraire les idéogrammes de leurs actualisations pour mettre en évidence ce qui, dans le fonctionnement sémiotique des caractères, le fascine. Il s'agit du rapport intrinsèque qu'entretiennent l'aspect visuel des signes et leur valeur signifiante, c'est-à-dire la relation texte-image contenue naturellement dans 
l'idéogramme. Le réalisateur explique ainsi sa démarche : «[...] d'une manière générale, nous avons par définition, dans le monde occidental, séparé les prérogatives du texte et celles de la peinture. L'une de mes récentes préoccupations a été de tenter de traiter ces deux éléments sous la forme d'un phénomène unique, en prenant comme archétype la notion de l'idéogramme oriental ${ }^{1}$ ». L'idéogramme soulève donc de nombreuses questions dans son rapport avec les composantes traditionnelles du cinéma, à savoir le scénario, le profilmique et l'image. Greenaway autonomise graduellement les diverses actualisations des livres composés par Nagiko sur des corps de jeunes hommes et des idéogrammes les uns par rapport aux autres. Les livres tels qu'ils ont été écrits en anglais avant le tournage (disponibles dans le scénario du film), ceux traduits en japonais et peints sur les corps des comédiens pour le tournage et ceux à nouveau traduits en anglais lors de la postproduction et banc-titrés à l'image, forment finalement trois entités relativement indépendantes qui certes, interagissent, mais ne sont pas le décalque les unes des autres. Ce principe disjonctif s'étend graduellement à tous les idéogrammes ostensiblement montrés à l'image. De sorte que la chronologie et le principe de causalité ne s'appliquent pas de la même manière pour la conduite narrative du scénario et pour les idéogrammes profilmiques. Ainsi, le Livre du séducteur écrit par Nagiko sur un jeune modèle offert à l'éditeur, comporte-t-il un paragraphe dans lequel est décrite, par avance, la situation physique du messager au moment où il sera lu :

Ceci est le corps bien lavé d'un livre,

Il se tient debout sur sa table ${ }^{2}$

Dans la séquence où l'éditeur découvre le contenu de ce huitième livre, le messager est trempé par l'averse qu'il vient d'essuyer, rendant la lecture partiellement impossible. Comme le prédit son propre contenu textuel, il a «le corps bien lavé » et se tient non pas debout, mais assis sur la table où l'éditeur vient de le projeter avec brutalité. Cette littéralisation du contenu textuel n'est possible que dans la mesure où la concaténation des éléments de scénario et le déploiement de ce que nous serions tentés d'appeler la sphère idéographique, n'appartiennent pas à la même chronologie. Il est en effet impossible que Nagiko ait pu prévoir les circonstances dans lesquelles serait lu le huitième livre. Elle les a pourtant décrites dans le texte même du livre.

24 A la chronologie réaliste se substitue une autre logique de coprésence ou de coïncidence transversale. Ainsi, les idéogrammes coïncident-ils souvent avec la situation qui les rend lisibles, indépendamment de tout réalisme. Dans la séquence où le jeune amant japonais calligraphie sur la poitrine de Nagiko, un léger décalage de la bande son et de l'image permet de faire coïncider le dialogue de la jeune femme "You're not in a good position to reach clean leaving》 avec l'inscription de 《乾淨》 à l'encre rouge. Le terme 乾淨 (signifiant littéralement " propre ») coïncide avec l'adjectif anglais « clean » employé par la jeune femme. De même, les idéogrammes 嚴禁 (strictement interdit) peints à l'encre noire sur les épaules de Nagiko sont en accord avec la séquestration dont la jeune femme est victime par des militants écologistes extrémistes. Rien cependant ne justifie la présence de ces idéogrammes, hormis une logique sémantique juxtadiégétique.

25 Les incessants changements de ratio pratiqués par le montage dans The Pillow Book convoquent à l'évidence le niveau plastique de l'image. Le choix du cadre est directement approprié aux motifs contenus dans le plan. En pratiquant ces variations incessantes, Greenaway se réapproprie les prérogatives du peintre à l'intérieur d'un objet, par ailleurs soumis à des normes de diffusion particulièrement contraignantes. Il n'hésite pas à sacrifier une partie de l'espace offert par le standard de pellicule choisi et redéfinit à 
l'envi un cadre approprié à chaque plan, ou presque. La dimension plastique de l'image, vue avant tout comme une surface plane sur laquelle s'inscrivent les niveaux de gris et des couleurs, est rendue constamment sensible. La conscience du spectateur est stimulée dans deux directions paradoxales et concurrentes : la dimension plastique et la dimension diégétique. Les multiples pages de livres ou de parchemins, billets, lettres et autres surfaces planes écrites qui sont affichées en plein écran, ne sont pas actualisées au sens habituel du terme. On les dirait plus en mention ou en exposition qu'en situation. Une part d'abstraction semble se saisir de ces grandes surfaces blanches sur lesquelles se déploie la sphère idéographique.

De fait, Greenaway oppose deux logiques. Au développement temporel du plan répond le statisme spatial de la surface. Les idéogrammes font alors office de chevilles, articulant les divers niveaux ontologiques de l'écran. Ils sont les objets cruciaux permettant le passage de l'abstrait au figuratif, du plastique au photographique. L'examen des surfacesécrans met en lumière les divers degrés d'iconicité ménagés par le réalisateur tout au long du film. La page blanche du journal de Nagiko est le modèle d'abstraction sur lequel se déploient les idéogrammes non figuratifs. La présence d'impressions sigillaires et de caractères d'imprimerie induisent déjà une forme d'iconicité. Ces traces d'une impression mécanique ou manuelle relèvent en partie de la trace ontologique. Elles désignent une action passée : quelque chose a pu s'imprimer là, sur cette surface jusqu'alors abstraite.

27 Sur les pages de son journal, Nagiko ne se contente pas de tracer les caractères selon une disposition fantaisiste. Elle organise ces derniers à la manière de la poésie figurative ${ }^{3}$, mimant par exemple la silhouette d'une colline et d'un astre rond surplombant. L'image formée par la page blanche comporte donc un niveau figuratif. Mais c'est le travail de transparence effectué lors du montage qui permet à l'image d'accéder au niveau diégétique à proprement parler. Le réalisateur construit un texte-image à trois niveaux. C'est en effet la section cent cinquante du Pillow Book de Sei Shonagon qui fait office de lien visuel entre la jeune Nagiko et son modèle spirituel, la courtisane de l'ère Heian ${ }^{4}$. Les visages des deux femmes se rapprochent l'un de l'autre jusqu'à se superposer totalement à mesure que la page calligraphiée s'estompe pour finalement disparaître. Le Pillow Book s'efface donc une fois les deux calligraphes réunies par l'image. Le texte-image intègre ici la dimension temporelle, car il évolue et son sens se forme dans la durée du plan.

28 Greenaway formalise plus encore le rapport du texte calligraphié et de l'image filmique dans les arrêts sur image qui miment la capture photographique de Nagiko par le jeune photographe Hoki. Chaque cliché est traité de manière très graphique: contrastée à l'extrême, jusqu'à ne plus offrir qu'une opposition de surfaces blanches et de surfaces noires, l'image est superposée en transparence avec une page du journal de la jeune femme. Les silhouettes de Nagiko et de l'avion de ligne, en approche pour l'atterrissage, sont comme dessinées à l'encre noire et au pinceau. Les matières des idéogrammes et de l'image photographique se confondent pour former une manière d'estampe épurée. On voit bien ainsi comment le réalisateur, en opérant un travail de confusion des matières et en figeant l'image, utilise l'idéogramme au profit de la plasticité de l'image photographique et filmique. L'écran devient alors en partie un espace plastique.

Dans le cinéma dominant, le noir est synonyme d'absence de signal, de pause dans la sémiose. Il est le plus souvent accompagné (sauf effet dramatique contraire) d'un silence de la bande son. Cette convention s'est établie de manière mimétique avec les données physiques de la photographie, la présence de lumière étant assimilée à l'existence d'un enregistrement, et donc d'un signe, alors que le noir est ressenti comme un état de veille, 
de pré-enregistrement. Mais, si les bandes noires du format letterbox télévisuel résultant d'un changement de format et de ratio, signalent un espace d'absence de lumière en logique additive, il est un fait que leur présence généralisée depuis une dizaine d'années répond à des motivations purement esthétiques. On peut y voir une recherche de distanciation référentielle délibérée et ironique vis à vis de la transformation que subit le film à son passage à la télévision, mais également et tout aussi sûrement, une manifestation de la capacité du média vidéo à récupérer la valorisation attachée au média cinéma. L'esthétisation graduelle du noir encadrant l'image a, sans aucun doute, joué un rôle facilitant dans la naturalisation des écrans multiples.

30 Le spectateur postmoderne connaît à présent les surfaces noires mises à jour par le compositing et sait qu'elles ne relèvent d'aucune nécessité technique. Il s'agit de fait d'un espace de construction, d'un repère sur lequel le collage va pouvoir s'effectuer. Certains outils informatiques nomment cette surface le «canevas». Le canevas serait donc cet espace disponible, bornée par le ratioet le format (en pixel) final recherchés, capable de recevoir les images dans un processus naturalisé à la fois de montage linéaire et de collage vertical.

31 La question reste cependant posée de la perception du canevas par le récepteur qui n'a pas accès aux étapes de la facture de l'image. A l'évidence, le cadre multiple s'inscrit dans une dialectique de la fragmentation et du flux et met en cause la notion de cadre-limite telle que définie par Aumont ${ }^{5}$. La manière de marie-louise formée par la zone de noir faitelle partie de l'image, ou bien en est-elle figuralement exclue pour former la représentation d'un dispositif à plusieurs écrans? La fragmentation de l'image en plusieurs points de vue s'oppose radicalement à la délimitation de l'espace définie par le tableau renaissant dans le cadre de la géométrie euclidienne. Ici, l'image n'a plus de centre et le canevas devient la surface plate délimitée par ses qualités plastiques. On trouve là une des définitions possibles du tableau de la modernité et, peut-être aussi, de ce que Pierre Francastel appelle l'« art objectif $^{6} »$, dans lequel l'auteur fait coexister des formes telles que l'art pariétal égyptien et le cubisme.

L'appropriation par les réalisateurs de l'ergonomie du canevas varie en fonction des objectifs dramatiques de la fiction. Ainsi, la série américaine 24 fait-elle massivement appel aux longues focales pour multiplier les gros plans sur les visages des protagonistes. La diminution de la profondeur de champ et la forme de planéité qu'elle induit opère un recentrement des vignettes ${ }^{7}$ sur les figures (ici les visages des personnages), excluant en partie la perspective. La thématique omniprésente de l'isolement individuel se nourrit de ce que les personnages sont représentés comme une succession d'îlots individuels disjoints bien qu'ils travaillent au sein d'une même cellule antiterroriste. La suspicion entretenue entre les protagonistes induit le paradoxe de l'isolement à l'intérieur d'une aspiration communautaire et patriotique forte.

Autre paradoxe, cette fois formel, de la série : on constate que la fragmentation s'avère être le vecteur de la constitution d'un flux visuel apparemment continu. Les cadrages multiples et changements incessants de points de vue dans le contexte d'une conversation téléphonique produisent en effet un sentiment de flux informatif là où un champ contre-champ eut amplement suffi. Dans son étonnant Counter Phrases, Thierry de May opère de multiples combinaisons d'écrans, en corrélation avec la danse, dans une volonté de mise en évidence du mouvement, au prix parfois de sa réduplication synchronique et diachronique. Le montage emploie de fait un grand nombre de procédés de composition, induisant la coprésence d'au moins deux fois le même plan en même 
temps : changements d'échelle, champs et contre-champs, doublons exact, isolements et mises en regard, variations d'angle, variations d'exposition et de mise au point, spécularités (par reflet dans les eaux d'un plan d'eau), panoramiques, etc. Là aussi, la multiplication des points de vue veut être ressentie comme la délivrance d'informations supplémentaires, alors qu'il ne s'agit que de répétition.

En guise de typologie minimale, on peut discriminer deux types d'utilisations particulièrement différentes des écrans multiples. La première relève de mécanismes ascendants. Elle privilégie une manière de grammaire efficace et non problématique au service du flux d'informations et de la sensation produite chez le spectateur. La seconde relève, au contraire, de mécanismes descendants. Elle exige une attention particulière portée à la composition et une interprétation sémantique de celle-ci. Probablement relève-t-elle du maniérisme selon Serge Daney: l'image l'emporte sur tout le reste et s'oppose à la notion de flux.

35 Dans l'optique que nous nommerons « grammaticale », la présence d'écrans multiples est instrumentalisée au profit de la gestion de l'espace ou du temps. Dans la première séquence de Phone Booth de Joel Schumacher, une gigantesque mosaïque se forme, faite de dizaines de gros plans sur des usagers du téléphone, signifiant l'omniprésence du réseau dans la ville. Ici, le lieu multiple est figuré par les écrans multiples. C'est d'ailleurs l'essentiel de l'utilisation faite par le film de la vignette : mettre en relation le lieu de celui qui appelle (l'image plein cadre) et le lieu de celui qui reçoit l'appel (la vignette). On est ici dans une situation équivalente à celle d'un split screen traditionnel.

Dans son Time Code, Mike Figgis fonde le contrat de lecture sur la règle du temps réel : les quatre vignettes de tailles équivalentes sont synchrones et chacune d'entre elles participe du plan séquence d'une heure et demie qui constitue le film. Il n'y a, de la première à la dernière minute, aucun choix de composition visuelle, et bien souvent, la bande son est le seul élément qui marque la synchronicité. La composition, toute entière au service de la narration, n'a pour seul but que la délivrance d'informations simultanées. Aucune intelligence visuelle ne vient au secours de la narration, sauf à considérer la coprésence de personnages géographiquement éloignés comme des marqueurs de théâtralité ressuscitant des figures oubliées par le cinéma comme les surdités opportunes, les apartés, etc. Mais là encore, les effets les plus marquants et les plus significatifs sont du ressort de l'opératique, et donc du sonore, puisque le réalisateur, lors des premières projections, effectuait le mixage des quatre pistes et de la musique en temps réel, allant jusqu'à jouer certaines parties de trompette pendant la projection. La notion de film concert est ici parfaitement opératoire et renvoie à la logique de flux et au sound design du cinéma postmoderne. On pense immanquablement aux sons artificiellement apposés aux mouvements, à l'apparition ou à la disparition des vignettes dans certains films à la recherche de la sensation la plus forte. Cette logique de « son et lumière » se fonde sur un détachement du son et du représenté (et donc sur une annulation de l'iconicité) de l'image au profit de la relation du son et de l'objet-vignette. Somme toute, le son matérialise et met en spectacle l'afflux des images et des informations, c'est-à-dire le montage lui-même.

37 A l'opposé de ce type de banalisation de l'écran multiple, Besenbahn de Dietmar offenhuber offre un excellent exemple de composition signifiante, car il traite le rapport espace-temps de manière purement visuelle. La multiplication du même plan de travelling latéral dont les huit ou neuf exemplaires sont disposés côte à côte mais décalés de quelques photogrammes reconstitue un continuum spatial tout en exhibant la 
continuité temporelle qui relie chacune des vignettes à ses voisines immédiates. Dans un registre plus métaphorique, on trouve dans Take care of my cat du Coréen Jae-eun Jeong des plans qui semblent dans un premier temps représenter un espace cohérent, mais se révèlent être, à la faveur du déplacement d'un protagoniste, un collage regroupant un champ et un contre-champ. Le réalisateur tisse ainsi un réseau de signification résonnant avec la gémellité de deux des personnages féminins du film. L'utilisation du collage filmique relève alors d'une écriture à part entière, et non plus de l'effet.

On peut d'ailleurs proposer d'étendre la notion de montage à l'étape du compositing: le montage, qui est en fait un collage diachronique, acquiert une dimension synchronique et voit le canevas s'instituer en surface spatio-temporelle vierge, capable de recevoir des matériaux dans ses deux dimensions. La notion de plan est largement mise à mal par le jeu des vignettes qui dialoguent dans l'espace du canevas, sans qu'aucune ne puisse prétendre jamais faire référence dans le domaine de la continuité temporelle ou spatiale. Bien que le montage ait toujours été une opération cruciale et créative, le compositing bénéficie d'une liberté bien plus grande, car il s'affranchit aisément des lois de la concaténation causale. On trouve cependant des réalisateurs pour effectuer un travail évident de structuration grammaticale de cet outil dans le cadre d'une visée narrative.

39 Cette « visual litteracy » qui, selon le réalisateur, manque cruellement au cinéma, trouve avec le compositing les moyens de sa pleine expression. En trois films, Greenaway met en place et propose une esthétique filmique capable non seulement de tenir la durée d'un long métrage, mais également de faire sens. Rivalisant avec les expérimentations du cinéma des premiers temps, il tente de structurer un vocabulaire visuel en usant massivement de procédés qui, dans le cinéma narratif contemporain, restent marginaux.

40 Dans Prospero's Books, une sémantique du composite est amorcée. D'une part les lignes de composition produites par le collage des vignettes sur les écrans cadres prennent souvent sens et éclairent ou soutiennent la situation dramatique du plan. D'autre part, la distribution des ces vignettes en fait, sur l'ensemble du film, un marqueur de virtuel. On y voit en effet soit le déploiement de la magie de Prospéro, souvent figurée de manière naïve, soit la vision prédictive du magicien, soit encore le point de vue omniscient qui caractérise le grand savant, soit enfin les vingt-trois livres magiques qui fondent son pouvoir. Il existe donc une manière de distribution grammaticale de l'emploi des vignettes, même si sa portée significative reste élémentaire car peu articulée. The Pillow Book, quant à lui, instaure et installe une forme de naturatisation de l'espace « canevas ». Le fond noir de l'écran n'apparait plus comme une option de la composition, mais une constante, une base active sur laquelle il convient de construire une image qui n'est jamais pré-donnée. Si, de rares fois, l'image se superpose bord à bord avec le canevas, c'est par effet consenti de réalisme, au sens ou le réalisme, qui considère la fenêtre de l'écran comme le mime du regard humain, est une idéologie en soi. Les images qui viennent se composer les unes aux autres sur la surface disponible du canevas conservent le ratio et la taille qui leur sont assignés par leur nature. Le format vertical de la feuille de papier A4 (parangon du volumen, le rouleau support d'écriture) s'oppose donc au format horizontal du billet de banque (apparenté quant à lui au 16/9 $9^{\text {ème }}$ de l'image cinématographique) sans que le format de l'écran n'intervienne dans la négociation. Ici le réalisateur semble s'être beaucoup moins intéressé aux possibilités sémantiques du composite. L'attention est bien plus portée sur le dialogue formel entre les divers formats d'images utilisés dans la vie courante (mais aussi dans les usages du cinéma), avec comme point de focalisation les diverses actualisations de l'idéogramme comme métaphore de la relation texte-image. Le 
cycle des Tulse Luper Suitcases, enfin, développe une manière de montage multidimensionnel qui active à la fois les mécanismes de la succession des images dans le temps, mais aussi ceux relatifs à leur coprésence et leur poly-présence. Ce sont ici les procédés synchroniques qui déclenchent la production de sens. La même image, montrée simultanément à divers stades de son défilement produit non seulement l'effet de sens relatif aux objets représentés qu'elle contient, les effets de suspens et d'anticipation du à la délivrance d'information diachroniques, mais aussi un effet cinétique particulier dans lequel la répétition magnifie le mouvement, le fait circuler dans l'espace du canevas et lui donne une nouvelle signification conditionnée par les paramètres du compositing. Si le procédé n'est pas nouveau, il revêt tout son intérêt grâce à la maîtrise du montage, la richesse et la justesse avec lesquelles ces divers procédés sont mariés en fonction de la dramaturgie. Les notions de plan, de séquence ou de fil narratif sont, bien entendu, largement perturbées, pour ne pas dire abolies.

41 Après avoir, durant plus de vingt ans, tenté d'amener la peinture au cinéma, Peter Greenaway réalise aujourd'hui l'opération inverse en projetant sur la célèbre Ronde de nuit de Rembrandt ou sur la Scène de da Vinci des images haute définition qui animent, mais surtout redessinent et mettent à jour la composition des tableaux originaux. Il ne s'agit plus là de composition, mais d'hybridité. Signe qu'une nouvelle ère s'ouvre aux réalisateurs qui désirent s'emparer des nouvelles technologies, les notions d'écran, de composite ou de cinéma expansé participent de la redéfinition d'un lectorat et de codes de représentations inédits.

\section{NOTES}

1. GREENAWAY, Peter, "Un abécédaire de la provocation ", Le grand atelier de Peter Greenaway, Université des sciences humaines de Strasbourg, Art \& université, Les presses du réel, Strasbourg, 1998, p.150

2. GREENAWAY, Peter, «Les treize livres écrits sur la peau, Le huitième livre. », The Pillow Book, Edition Dis Voir, Paris, p. 109.

3. La poésie figurative, dont l'invention est généralement attribuée à Simmias de Rhodes $(\sim \mathrm{IV}$ s.) inclut les pratiques modernes telles que le calligramme d'Apollinaire ou les inventions plastiques de Mallarmé (Un coup de dé jamais n'abolira le hasard).

4. $\mathrm{X}^{\mathrm{e}}$ siècle.

5. AUMONT Jacques, L'image, Nathan, 1990

6. FRANCASTEL Pierre, La réalité figurative, 1965

7. Nous emploierons le terme «vignette» pour désigner les éléments constitutifs d'un écran multiple. 


\section{RÉSUMÉS}

Approche esthétique et ergonomique de l'influence des outils numériques sur la création cinématographique à caractère pictural dans les œuvres de Peter Greenaway.

Aesthetic and technical approach of digital image making and its influence on cinema in Peter Greenaway's films.

INDEX

Mots-clés : cinéma, hybridité, numérique, Peter Greenaway, pictural

Index géographique : Europe

Index chronologique : XXIe siècle

\section{AUTEUR}

FABIEN MAHEU

Ecole Européenne Supérieure de l'Image 